\title{
Appendix C
}

\section{Excerpt from an Unedited Volume of $L e$ Roman de Perceforest, MS Bibl. Nat. fr. 346}

[The knight Lyonnel has, through the treachery of an enemy knight, lost the trophies that would have enabled him to win his beloved; he has also lost contact with the lady herself, a fairy. Riding along aimlessly in the wilderness, he engages in a lengthy lament.]

[fol. 234] “. . . Et combien que soye destourbé oultre mesure, sy feray je ung lay par quoy en aucun temps sçaront les amoureus ma mescheance." Adont se teut l'espace de iiii. lieucs anglesches sans soy mouvoir ne mot dire. Mais aprés commença son lay en telle maniere qui s'ensuyt.

[The lay recounts Lyonnel's victories over a lion, a serpent, and a giant, his subsequent loss of the trophies of these battles, and his great sorrow. See Lods ed., Les Pièces lyriques du roman de Perceforest.

[fol. 235v] Quant Lyonnel eut son lay parfurny ainsi que cy dessus avez oÿ, il leva la teste et dist tout en hault, "Or vouldroie je que tous amans par amours sceussent mon lay. Sy savoient partie de mon meschief. Car il ne porroit estre qu'ilz n'eussent aucune pitié de moy; si prieroient pour moy." Tandiz qu'il disoit ces parolles, il oyt ung homme toussir par derriere luy. Lors tourna son viaire par devers luy et veyt que c'estoit ainsi comme il luy fut advis ung menestrel de la harpe. Sy luy demanda, "Sire varlet, estez vous menestrel?"- "Sire, dist il, oÿl." Adont luy demanda Lyonnel dont il venoit si prez de luy et il luy dist qu'il s'en aloit vers Bretaigne pour estre a la feste du Roy Percheforest. Mais quant il s'embaty sur luy et il le veyt ainsi embronchié, il s'aresta pour veoir a quelle fin il estoit en tel point. - "Or vous ay, sire, oÿ dire que vous avez fait ung lay que vous vouldriez que tous amans sceussent. Par ma foy se vous le me vouliez aprendre, je le joueroie encores en maint lieu."

"Varlet, dist Lyonnel, je le te diray voulentiers. Mais il n'a point de chant. Et se tu luy vouloies faire ung chant piteux selon le dit, je t'en sçaroie bon gré." - "Par ma foy, sire, [fol. 236] dist le menestrel, je le 
feray volentiers." Adont luy dist Lyonnel le lay tant de fois qu'il le sceut par coeur. Aprés ce ala attaindre le menestrel sa harpe et ala en pou d'heure faire dessus ung chant si piteux que quant Lyonnel luy oyt harper et chanter si piteusement le coeur luy fondy, tout en larmes, et fut si destraint qu'il ne se peult soustenir. Ainçois s'embroncha sur ses mains une grant piece, de la grant douleur qu'il sentoit en son coeur. Et quant le menestrel le veyt en tel point, il en eut pitié et dist, "Ha! sire chevalier, confortez vous et ne menez tel dueil. Car il n'affiert pas a chevalier de la renommee dont vous estes selon ce que vostre lay dist. Car vous estes celluy qui a mis a mort le lyon et le serpent et le gueant aux cheveulx dorez. Mais cueilliez coeur et cerchiez tant que vous sachiez qui vous a ainsi desnué."

Quant Lyonnel entendy le menestrel, il luy dist, "Par ma foy, ainsi le feray que tu m'as conseillié." Lors monta et prist congié a luy et se mist au chemin et le menestrel demoura tout seul. Si prist sa harpe et la mist en son estuy, et puis se mist au chemin et ala tant qu'il se trouva au pilier Estonné. Lors s'assist emprés et prist sa harpe, et commença a harper le dit si hault et si bien que c'estoit une pitié a oÿr. Mais quant il eut harpé, il ne garda l'heure qu'ils s'embatirent sur luy .iii. jeunes damoiselles vestues de blanches vestures si noblement que ce sambloient deesses ou faees. Adont dist l'une des damoiselles au menestrel, "Dy moy, par amours, qui fist ce lay et qui te l'apprist?" - "Dame, dist il, il le m'aprist celluy qui le fist. Et celluy qui le fist est le chevalier a qui le fait est advenu que le lay devise." - "Par ta foy, dist la damoiselle, scez tu ou le chevalier est?" - "Par ma foy, dame, dist il, je ne sçay fors tant qu'il va querant confort de sa douleur." - "Sire menestrel, dist la damoiselle, il convient que vous venez avecques nous. Sy nous aprendrez le lay." - "Dame, [fol. 236v] dist il, je le feray voulentiers." Mais ore se taist l'ystoire a parler d'eulx.

[After an interlude focusing on other characters in the romance, the story line returns to the minstrel, whom the three maidens lead before the king.]

[fol. 242v] Quant le menestrel eut joué devant le roy, il s'en vint seoir par devers les pucelles. Car Blanche desiroit moult de sçavoir le lay de la complainte. Sy fist tant qu'elle le sceut de point en point. Cy vous dy pour certain qu'elle fut si courroucee et a tel meschief de la mesaise qu'elle sçavoit que le bon chevalier souffroit pour elle, et pour la perte qu'il avoit faicte pour le chief du gueant qu'elle ne sçavoit qu'elle peust devenir. Mais en la fin s'advisa qu'elle feroit ung lay pour resconforter le chevalier. Car autrement ne sçavoit trouver voie de luy aidier. Cy ne fina toute la nuyt de penser tant qu'elle eut finé son lay a son vouloir. Et sy tost qu'elle fut levee la matinee, elle l'aprist au menestrel et luy pria moult qu'il le jouast par tout tant que le chevalier desconforté le sceust. - "Certes, madamoiselle, dist il, je le feray. Car je ne cesseray tant que je l'auray trouvé. Mais dictes moy qui diray je qui ce lay luy envoie?" [fol. 
243] - "Menestrel, dist la damoiselle, se vous trouvez le chevalier, dictes luy que la pucelle qu'il veyt baignier en l'escault l'a fait pour le resconforter. Mais hastez vous de vostre chemin, car tart m'est qu'il le sache." - "Damoiselle, dist le menestrel, je vouldroie estre au chemin." Adont le fist la damoiselle mengier et puis le mist hors du manoir et se trouva au pilier ou les pucelles l'avoient trouvé jouant son lay. Lors se mist au chemin et erra plusieurs journees que oncques ne peult oÿr nouvelles de Lyonnel. Et sachiez que en plusieurs lieux joua le lay ou il y avoit dames et chevaliers qui le lay moult prisierent, car moult doulcement confortoit la pucelle le chevalier.

Ung jour avoit le menestrel erré en la haulte forest jusques a la nuyt, adont s'aresta au pié d'une roche et s'apensa qu'il demourroit la endroit jusques a lendemain. Quant il eut la esté assiz une grant piece, il prist sa harpe et commença a jouer plusieurs choses. Et aprés prist a jouer le lay que Blanchete avoit fait qui estoit tel.

[The lay instructs Lyonnel to seek the temple where his lost trophies are being kept for him. See Lods ed., Pièces lyriques.]

[fol. 244] Tandiz que le menestrel se deduisoit en chantant son lay, il y avoit assez pres de luy ung chevalier qui s'estoit tapy en [fol. 244v] une roche qui avoit oÿ chanter le lay au menestrel. Dont s'esmerveilloit moult dont tel lay venoit. Lors s'apparut au menestrel dont il estoit pres et luy dist, "Sire, par amours, dictez moi qui fist ce lay que cy avez chanté si doulcement." - "Sire, dist le menestrel, je ne sçay qui vous estes, mais au dit le povez vous sçavoir puis que l'avez ö̈." - "Ha! gentil homme, dist le chevalier, je ne le demande pour nul mal, mais il semble qu'il ait esté fait aprés ung lay que je fiz n'a pas ung moys pour ung meschief qui m'estoit advenu n'a pas longtemps. Sy l'appellay le lay de la complainte." - "Comment, sire chevalier, dist le menestrel, estes vous celluy qui feïstes le lay de la complainte?" - "Par ma foy, sire, dist il, oÿl. Ce suy je qui languiz et meurs de paour, sy que a pou ne cheÿ en desespoir de jour en jour."

"Sire, dist le menestrel, oncques ne cheÿ si bien a chevalier de lay qu'il feist comme il a fait a vous." Lors luy compta comment les .iii. pucelles l'escouterent tandiz qu'il le jouoit dessoubz le pilier Estonné, et comment elles l'emmenerent en leur manoir. Et puis luy compta comment la plus jeune des pucelles le requist qu'il luy aprist le lay. - "Et en celle nuyt mesme, la pucelle en fist ung autre et le m'aprist le lendemain et me pria que je le jouasse tant que le chevalier qui fist le lay de la complainte l'eüst oÿ pour soy resconforter. Cy m'en est bien cheü que vous m'avez trouvé le lay jouant. Et sachiez que la pucelle qui ce lay a fait est celle que vous veïstez baignier en l'escault. Et vous mande qu'elle l'a fait pour vous resconforter." - "Ha! gentil homme, dist le chevalier, je te prie que tu le vueillez jouer encore une fois tant que l'aye bien entendu." - "Sire, dist le menestrel, je le feray volentiers."

Adont commença le menestrel a jouer le lay moult piteusement. Cy 
Appendix C

devez sçavoir que tandiz qu'il le jouoit le chevalier jectoit aucunes foiz si griefs souspirs que [fol. 245] c'estoit pitié a oïr. Et sy luy filoient les larmes des yeulx aussi grosses que poix de la grant joye qu'il avoit au coeur. Mais quant le menestrel eut le lay joué le chevalier ala dire, "Certes, Sire, grandement m'avez servy a gré, et benoiste soit la pucelle qui si bien m'a resconforté par sa doulce pitié. Mais par amours me sçairiez vous mener ou elle demeure?" - "Par ma foy, sire, dist le menestrel, nennyl, car elle demeure en faerie. Mais selon le lay qu'elle a fait il fault que vous querez le temple a la franche garde. Car la recouvrerez vous vostre perte." - "Par ma foy, menestrel, dist le chevalier, vous dictes voir. Mais dictes moy, me sçairiez vous enseigner la voie?" — "Par ma foy, sire, dist il, je ne sçay."

[At this point the minstrel and the knight, finally named as Lyonnel at the end of the episode, part, and the story moves on for a time to other adventures. Eventually, Lyonnel succeeds in finding the temple described in the lay, recovers his trophies, and is reunited with Blanche.] 